\title{
Crystal Structure of the $\alpha$-Form of Syndiotactic Polystyrene
}

\author{
C. De Rosa, G. Guerra, V. Petraccone, and P. Corradini \\ Dipartimento di Chimica, Università di Napoli, \\ Via Mezzocannone 4,80134 Napoli, Italy
}

(Received March 9, 1991)

\begin{abstract}
Models for chain packing for ordered and disordered modifications of the $\alpha$-form of syndiotactic polystyrene are suggested, through quantitative comparisons between X-ray diffraction intensities and calculated structure factors. According to the reported analysis, zig-zag planar chains are packed in the hexagonal unit cell proposed by Greis et al., but with different orientation and relative height of the phenyl groups. In particular, the phenyl groups in both ordered and disordered modifications are packed according to rhombohedral symmetry. The disorder in the $\alpha$ modification corresponds to the statistical occurrence of two different, nearly isosteric, orientations of triplets of chains at well defined locations of a threedimensional lattice, which generate a rhombohedral symmetry for the whole unit cell.

KEY WORDS Syndiotactic Polystyrene / $\alpha$-Form / Crystal Structure / Order-Disorder Phenomena /
\end{abstract}

The synthesis of highly stereoregular syndiotactic polystyrene (s-PS) has been reported recently. ${ }^{1-3}$ The polymer presents very complex polymorphic behaviour. Three different crystalline forms and clathrate structures have been described and studied essentially by $\mathrm{X}$ ray diffraction, ${ }^{4-13}$ electron diffraction, ${ }^{14-16}$ FTIR, ${ }^{17-20}$ solid state NMR, ${ }^{23,24}$ and conformational energy analyses. ${ }^{25,26}$

Following the nomenclature proposed in ref 9 , the two crystalline forms $\alpha$ and $\beta$ contain planar zig-zag chains (line repetition group $\mathrm{t} c$ and conformation (TTTT) $)_{n}$ ) with an identity period $c=5.1 \AA$, while the third one (the $\gamma$ form), as well as the clathrate structure ( $\delta$-form) contain helical chains (line repetition group $\mathrm{s}(2 / 1) 2$ and conformation $\left(\mathrm{TTG}^{-} \mathrm{G}^{-}\right)_{2}$ or $\left.\left(\mathrm{G}^{+} \mathrm{G}^{+} \mathrm{TT}\right)_{2}\right)$ with identity period $c=$ $7.8 \AA$.

Conformational energy calculations, performed at our laboratories, ${ }^{26}$ indicate that the conformations $t c$ and $s(2 / 1) 2$ have nearly the same energy.

The general pattern is further complicated by the fact that both the $\alpha$ and $\beta$ forms (characterized by the same tc chain conformation and different chain packing) can exist in different modifications having different degrees of structural order, so that two limiting disordered modifications (the $\alpha^{\prime}$ and $\beta^{\prime}$ ) and two limiting ordered modifications ( $\alpha^{\prime \prime}$ and $\left.\beta^{\prime \prime}\right)$ have been described. ${ }^{9}$

In a recent paper $^{12}$ we proposed detailed models for the chain packing for the limiting $\beta^{\prime}$ and $\beta^{\prime \prime}$ modifications, through quantitative comparisons between X-ray diffraction intensities and calculated structure factors; furthermore it was shown that the disorder present in the $\beta$ form could correspond to a statistical occurrence of two different kinds of stacking of ordered bilayers of macromolecules.

As far as the $\alpha$ form is concerned, a detailed description of the morphology of thin films by transmission electron microscopy has been given, ${ }^{14-16}$ Greis et al. ${ }^{14,15}$ have proposed a hexagonal unit cell with $a=26.26 \AA$, in which clusters of three chains are packed according 
to the space group $\mathrm{P} \overline{6} 2 c$.

In a previous paper, ${ }^{9}$ we have shown, on the basis of systematic $\left(\alpha^{\prime}\right)$ and quasi-systematic $\left(\alpha^{\prime \prime}\right)$ extinctions, that the phenyl groups must satisfy a rhombohedral mode of packing and, thus, alternative models for the molecular packing in those modifications must be proposed.

In this paper these models for the $\alpha^{\prime}$ and $\alpha^{\prime \prime}$ modifications are described in detail and quantitative comparisons between $\mathrm{X}$-ray diffraction intensities and calculated structure factors are presented.

\section{EXPERIMENTAL}

s-PS $\left(M_{w}=6.6 \times 10^{5}\right.$ determined by GPC) was supplied by Himont Italia. The polymer fraction insoluble in methyl ethyl ketone is $93 \%$.

Ordered $\alpha^{\prime \prime}$ modifications were obtained by melt crystallization process, conducted in a moulding press. $^{9}$ The polymer powders, initially in $\gamma$ form, were melted at $\approx 280^{\circ} \mathrm{C}$ and then slowly cooled to room temperature. Disordered $\alpha^{\prime}$ modifications were obtained by annealing at $\approx 200^{\circ} \mathrm{C}$ of quenched amorphous samples. ${ }^{9}$

Wide angle X-ray diffraction patterns were obtained with nickel filtered $\mathrm{Cu}-K \alpha$ radiation with an automatic Philips powder diffractometer.

Observed structure factors $\left(F_{\mathrm{o}}\right)$ have been obtained as the square roots of the experimental intensities corrected by $L P=(1+$ $\left.\cos ^{2} 2 \theta\right) / \sin ^{2} \theta \cdot \cos \theta, F_{\mathrm{o}}=(I / L P)^{1 / 2}$. The experimental intensities have been evaluated by measuring the area of the peaks in the X-ray powder diffraction patterns, after substraction of the amorphous halo.

Calculated structure factors $\left(F_{\mathrm{c}}\right)$ have been obtained as $F_{\mathrm{c}}=\left(\Sigma\left|F_{\mathrm{i}}\right|^{2} \cdot M_{\mathrm{i}}\right)^{1 / 2}$, where $M_{\mathrm{i}}$ is the multiplicity factor and the summation is taken over all reflections included in the $2 \theta$ range of the corresponding observed reflection peak. Only the values greater than the observable limit are reported. We assumed an isotropic thermal factor $B=8 \AA^{2}$ and the atomic scattering factors as in ref 27.

The value of $R^{\prime}=\left(\Sigma\left|F_{\mathrm{o}}-F_{\mathrm{c}}\right|\right) / \Sigma F_{\mathrm{o}}$ for the observed and unobserved reflections, as grouped in Table II up to $d=2.50 \AA$, is $11 \%$.

\section{RESULTS AND DISCUSSION}

The X-ray powder diffraction patterns of s-PS samples in an ordered modification, close to the limiting $\alpha^{\prime \prime}$ modification, and in a disordered modification, close to the limiting $\alpha^{\prime}$ modification, are reproduced in Figures 1A and $\mathrm{B}$, respectively.

The reflections observed in the spectrum of the $\alpha^{\prime \prime}$ modification are all accounted for by the hexagonal unit cell with axes $a=26.26 \AA$, $c=5.04 \AA$, proposed by Greis et al. ${ }^{15}$ In this structure, in view of the hexagonal diffraction geometry, chains with zig-zag planar conformation would be assembled in triplets as shown in Figure 2A. A rotation of $60^{\circ}$ and translation of $1 / 2 c$ applied to the cluster of Figure $2 \mathrm{~A}$ give rise to the cluster of Figure 2B. ${ }^{9}$ Greis et al. ${ }^{15}$ observed that these two triplets of chains have the same steric hind-

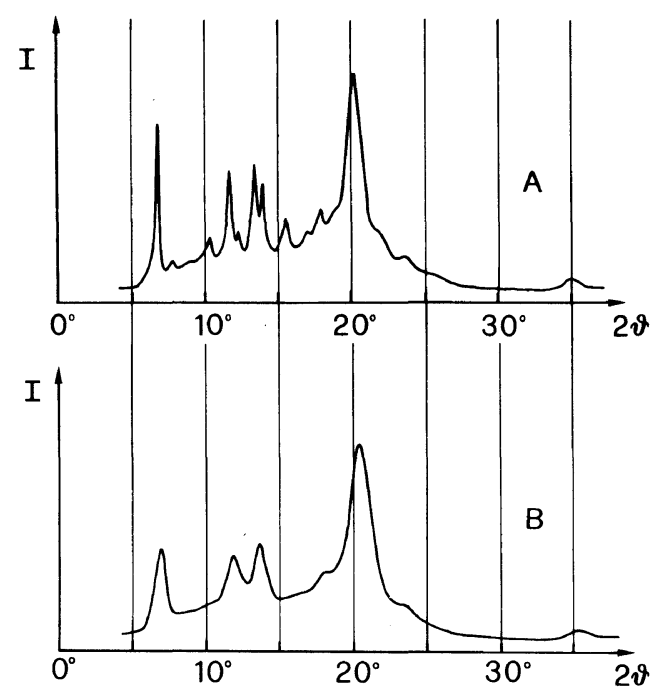

Figure 1. X-Ray diffraction patterns $(\mathrm{Cu}-K \alpha)$ of the $\alpha^{\prime \prime}$ (A) and $\alpha^{\prime}$ (B) modifications of s-PS. 
Crystal Structure of the $\alpha$-Form of Syndiotactic Polystyrene

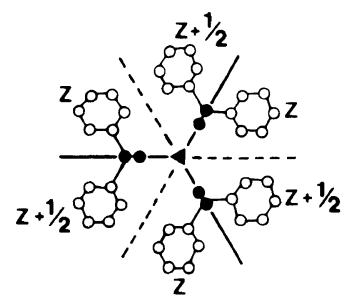

A

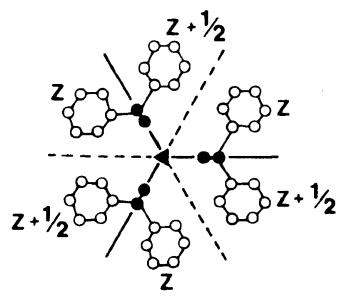

B

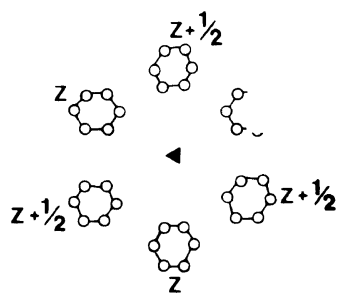

C

Figure 2. (A-B) Two different and isosteric orientations of triplets of s-PS trans planar chains. Both triplets $\mathrm{A}$ and $\mathrm{B}$ present a steric hindrance similar to that of the group of six benzene rings shown in $\mathrm{C}$.
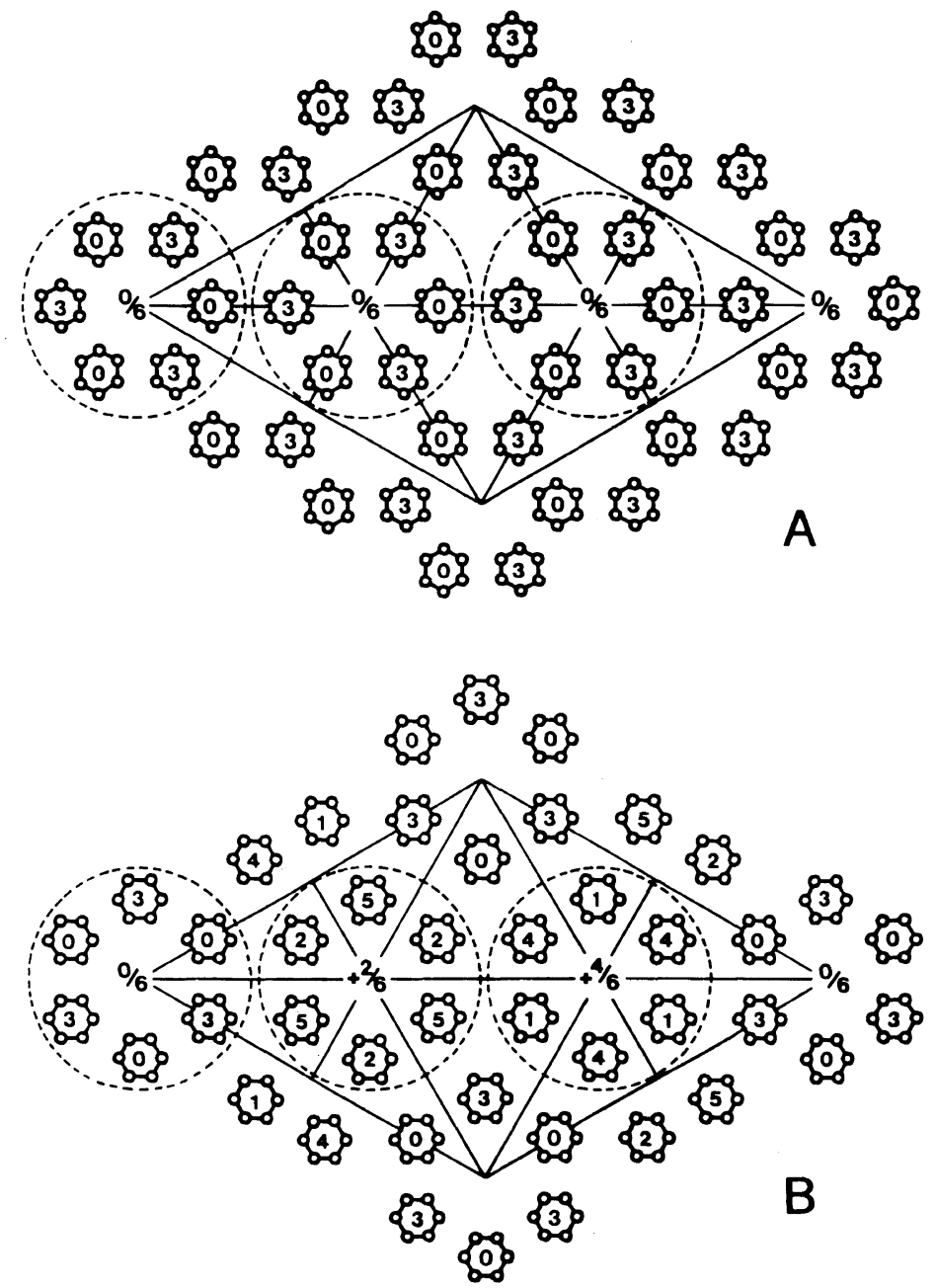

Figure 3. Models of packing of phenyl rings (relative height of the centers in units $c / 6$ ), proposed for the structure of the $\alpha$ form of s-PS A) by Greis et al. ${ }^{15}$ where the sextets of phenyl rings (corresponding to triplets of trans planar chains, see Figure 2), enclosed in dashed circles, are at a same height; B) in the present paper and having a rhombohedral symmetry. The sextets of phenyl rings, enclosed in adjacent dashed circles, present relative displacement of $2 c / 6$. 
rance in a unit cell, due essentially to six benzene rings (taking in case $\mathrm{A}$ and $\mathrm{B}$ almost identical positions), as shown in Figure 2C.

A close packing of these triplets can be achieved according to the models (referred only to the phenyl rings) shown in Figures $3 \mathrm{~A}$ and $3 \mathrm{~B}$. In both figures, three clusters of six phenyl rings are enclosed in dashed circles. In case A (hexagonal mode of packing) the three indicated clusters are at the same height; in case B (rhombohedral mode of packing) the three clusters are shifted one with respect to the other by $1 / 3 \mathrm{c}$. According to the following considerations, this last mode of packing is the correct one.

The intensities and Bragg distances observed in the X-ray powder diffraction patterns of the $\alpha^{\prime}$ and $\alpha^{\prime \prime}$ modifications are compared in Table I to the distances calculated according to the unit cell proposed by Greis et al. ${ }^{15} \mathrm{~A}$ large part of these data was already presented in Table I of ref 9 . It is possible to observe the systematic absence, in the $\alpha^{\prime}$ modification, of the $h k l$ reflections with $-h+k+l \neq 3 n$. These

Table I. Intensities and Bragg distances observed in the $\mathrm{X}$-ray powder diffraction spectra for the two $\alpha$ modifications ( $\alpha^{\prime}$ and $\left.\alpha^{\prime \prime}\right)$ of s-PS compared with the Bragg distances calculated according to the unit cell proposed by Greis et al. ${ }^{14,15}$

\begin{tabular}{|c|c|c|c|c|c|}
\hline \multirow{2}{*}{$h k l$} & \multirow{2}{*}{$d_{\text {cale }}$} & \multicolumn{2}{|c|}{$\alpha^{\prime}$} & \multicolumn{2}{|c|}{$\alpha^{\prime \prime}$} \\
\hline & & $d_{\mathrm{obs}}$ & $I_{\mathrm{obs}}$ & $d_{\mathrm{obs}}$ & $I_{\mathrm{obs}}$ \\
\hline 110 & 13.13 & 13.07 & $\mathrm{~ms}$ & 13.11 & $\mathrm{~s}$ \\
\hline 200 & 11.37 & & & 11.37 & $w$ \\
\hline 210 & 8.595 & & & 8.56 & $\mathrm{mw}$ \\
\hline 300 & 7.580 & 7.51 & $\mathrm{~m}$ & 7.58 & $\mathrm{~ms}$ \\
\hline 220 & 6.565 & 6.53 & $\mathrm{~m}$ & 6.56 & $\mathrm{~ms}$ \\
\hline 310 & 6.307 & & & 6.31 & $\mathrm{~ms}$ \\
\hline 400 & 5.685 & & & 5.67 & $\mathrm{~m}$ \\
\hline 320 & 5.217 & & & 5.18 & vw \\
\hline 410 & 4.962 & 4.94 & $\mathrm{w}$ & 4.94 & $\mathrm{mw}$ \\
\hline 211 & 4.347 & 4.34 & vs & 4.36 & vs \\
\hline 510 & 4.084 & & & 4.01 & vw \\
\hline 600 & 3.790 & 3.77 & w & 3.74 & $\mathrm{w}$ \\
\hline 610 & 3.468 & & & 3.50 & $w$ \\
\hline $10 \overline{2}$ & 2.505 & 2.55 & $\mathrm{mw}$ & 2.54 & $\mathrm{mw}$ \\
\hline
\end{tabular}

reflections are present, but with weak intensity, in the X-ray diffraction pattern of the $\alpha^{\prime \prime}$ modification.

This is an indication of the presence of rhombohedral symmetry for the $\alpha^{\prime}$ modification and a nearly rhombohedral symmetry for the $\alpha^{\prime \prime}$ modification. This suggests a packing of the phenyl groups as shown in Figure 3B, where adjacent clusters (along the $a-b$ direction) of six benzene rings (inside the dashed circles) are shifted along $c$ by $1 / 3 c$.

A good mode of packing among clusters of six phenyl rings (enclosed in the dashed circles) is achieved with the represented orientation (Figure 3B). The orientation differs with respect to the model preliminarily assumed as plausible by Greis et al. ${ }^{15}$ (Figure $3 \mathrm{~A})$ because of a $30^{\circ}$ rotation. This orientation assures, in this case, good Van der Waals contact distances between all benzene rings in the unit cell. For the unit cell of s-PS good Van der Waals contact distances are also assured if triplets of chains, of the kind A or B of Figure 2, are arranged inside each circle.

We think that statistical disorder between the two isosteric orientations of triplets of chains, shown in Figures 2A, B, could be present in the crystals of disordered $\alpha^{\prime}$ modifications. The position of the substituent phenyl rings remains substantially unaltered (that of Figure 3B), while the atoms of the polymer backbones are statistically distributed in six positions around the threefold axes. The structure can be described by the space group $\mathrm{R} \overline{3} c$.

In the ordered modification, the presence of $h k l$ reflections with $-h+k+l \neq 3 n$ (and their weakness) suggests that the packing of the phenyl rings could maintain a rhombohedral symmetry, while an ordering process of the positions of the polymer backbones could be involved.

Greis et $a .^{15}$ have already suggested that, for the unit cell of the ordered structure (containing three triplets), one triplet is oriented in one way and the other two in the 


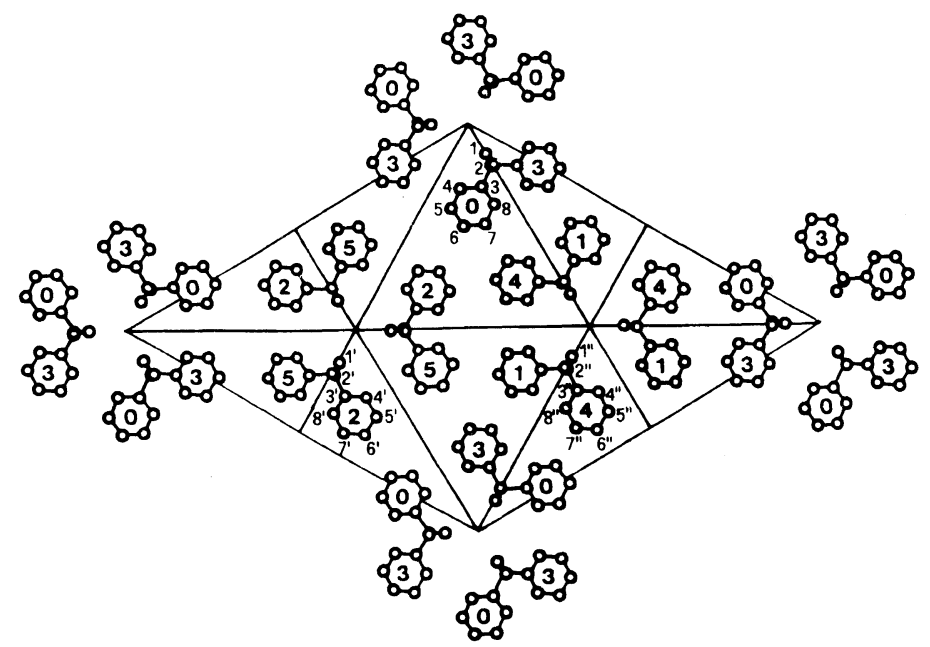

Figure 4. Possible model of packing for the ordered $\alpha^{\prime \prime}$ modification of s-PS. Relative heights of the centers of the phenyl rings are in units $c / 6$.

opposite way of Figure 2. The same assumption for our model of Figure 3B leads to the structure shown in Figure 4. In this model, only the atoms of the polymer backbones would not satisfy the rhombohedral symmetry and this is in agreement with the presence, as well as the weakness, of the reflections $h k l$ with $-h+k+$ $l \neq 3 n$. The space group for this model would be $\mathrm{P} 3 c 1$.

A comparison between the observed structure factors $\left(F_{\mathrm{o}}=(I / L P)^{1 / 2}\right)$, obtained from the $\mathrm{X}$-ray powder diffration patterns of Figures $1 \mathrm{~A}$ and $\mathrm{B}$, and those calculated $\left(F_{\mathrm{c}}=\left(\Sigma \mid F_{\mathrm{i}}\right)^{2}\right.$. $\left.M_{\mathrm{i}}\right)^{1 / 2}$ ) for the model of Figure 4, space group P3c1 ( $\alpha^{\prime \prime}$ modification) and for the statistical space group $\mathrm{R} \overline{3} c$ ( $\alpha^{\prime}$ modification) is shown in Figures 5A and B. For the $\alpha^{\prime \prime}$ modification (space group $\mathrm{P} 3 c 1$ ) a numerical comparison is also presented (Table II).

Fairly good agreement between observed and calculated intensities is obtained.

For the ordered modification (space group P3c1) the calculations of structure factors were performed with the coordinates of the asymmetric unit shown in Table III. The asymmetric unit corresponds to the three independent monomeric units labelled in Figure 4.
Given a set of coordinates $x, y, z$ of one monomeric unit (atoms $1-8$, Figure 4), the set of coordinates of the second independent unit (atoms 1'-8', Figure 4) are obtained by changing every $x, y, z$ of the first unit into $y+2 / 3, x+1 / 3, z+1 / 3$ (reflection at the plane 110 , through the origin, plus rhombohedral translation of $2 / 3,1 / 3,1 / 3)$. The set of coordinates of the third independent unit (atoms $1^{\prime \prime}-8^{\prime \prime}$, Figure 4) are obtained by changing every $x, y, z$ of the second unit into $x+2 / 3, y+1 / 3, z+1 / 3$ (rhombohedral translation of $2 / 3,1 / 3,1 / 3)$.

The described operation of reflection, for the particular coordinates as reported, generates from carbon atoms of one benzene rings in $x$, $y, z$ (atoms 3-8, Figure 4) a set of carbon atoms in $y, x, z$ with almost the same space occupancy.

For the statistical space group $\mathrm{R} \overline{3} c$ the asymmetric unit corresponds to one monomeric unit. The set of equivalent positions given for the $\mathrm{R} \overline{3} c$ group in the International Tables for X-ray Crystallography ${ }^{29}$ (36- $\left.f\right)$, may be also obtained starting from the coordinates in Table III, given for the space group $\mathrm{P} 3 c 1$ (equivalent positions 6- $d$ in International Tables $\left.{ }^{29}\right)$, and adding inversion centers at $(0$, 
Table II. Comparison between observed $F_{\mathrm{o}}$ and calculated $F_{\mathrm{c}}$ structure factors for the $\alpha^{\prime \prime}$ modification ( $\mathrm{P} 3 c 1$ space group)

\begin{tabular}{|c|c|c|c|c|}
\hline$h k l$ & $d_{\text {calc }}$ & $d_{\mathrm{obs}}$ & $\begin{array}{c}F_{\mathrm{o}}= \\
(I / L P)^{1 / 2}\end{array}$ & $\begin{array}{c}F_{\mathrm{c}}= \\
\left(\Sigma\left|F_{\mathrm{i}}\right|^{2} \cdot M_{\mathrm{i}}\right)^{1 / 2}\end{array}$ \\
\hline 110 & 13.13 & 13.11 & 166 & 172 \\
\hline 200 & 11.37 & 11.37 & 50 & 118 \\
\hline 210 & 8.595 & 8.56 & 120 & 132 \\
\hline 300 & 7.580 & 7.58 & 200 & 160 \\
\hline 220 & 6.565 & 6.56 & 326 & 371 \\
\hline 310 & 6.307 & 6.31 & 250 & 206 \\
\hline 400 & 5.685 & 5.67 & 210 & 201 \\
\hline 320 & 5.217 & 5.18 & 110 & 79 \\
\hline 410 & 4.962 & 4.94 & 234 & 192 \\
\hline$[500$ & 4.548 & & & 2057 \\
\hline 330 & 4.377 & 4.36 & 932 & $205\} 940$ \\
\hline 211 & 4.347 & & & $894]$ \\
\hline$\left\{\begin{array}{l}510 \\
31 \overline{1}\end{array}\right.$ & $\begin{array}{l}4.084 \\
3.937\end{array}$ & 4.01 & 444 & 435 \\
\hline$\left\{\begin{array}{l}600 \\
520\end{array}\right.$ & $\begin{array}{l}3.790 \\
3.642\end{array}$ & 3.74 & 300 & 220 \\
\hline 610 & 3.468 & 3.50 & 180 & 194 \\
\hline 440 & 3.283 & - & n.o. $(109)^{a}$ & 70 \\
\hline $42 \overline{1}$ & 3.270 & - & n.o. $(110)^{a}$ & 113 \\
\hline$\left\{\begin{array}{l}530 \\
700\end{array}\right.$ & $\begin{array}{l}3.249 \\
3.249\end{array}$ & - & n.o. $(111)^{a}$ & 117 \\
\hline 620 & 3.154 & - & n.o. $(114)^{\mathrm{a}}$ & 118 \\
\hline 630 & 2.865 & - & n.o. $(127)^{\mathrm{a}}$ & 110 \\
\hline 621 & 2.674 & - & n.o. $(137)^{a}$ & 137 \\
\hline$\left\{\begin{array}{l}900 \\
10 \overline{2}\end{array}\right.$ & $\begin{array}{l}2.527 \\
2.505\end{array}$ & 2.54 & 446 & 449 \\
\hline
\end{tabular}

a Reflections not observed (n.o.) in the Geiger spectrum. The numbers in parentheses represent values of $F_{\mathrm{o}}$ corresponding to threshold intensities, taken as equal to $1 / 2$ of the minimum observed.

$0,0),(2 / 3,1 / 3,1 / 3),(1 / 3,2 / 3,2 / 3)$.

The phenyl rings generated by the inversion centers remain substantially at the same positions they were in the ordered structure, as already shown for the triplets of chains of Figure 2. The calculated intensities of the reflections with $-h+k+l=3 n$ are thus very close to those calculated for the ordered
Table III. Fractional coordinates of the carbon atoms of the asymmetric units for the model of Figure 4 (space group $\mathrm{P} 3 \mathrm{c}$ ) ).

The asymmetric unit corresponds to the three independent monomeric unit labelled in Figure 4

\begin{tabular}{llll}
\hline & $x$ & $y$ & $z$ \\
\hline 1 & 0.051 & 0.102 & 0.000 \\
2 & 0.073 & 0.139 & 0.252 \\
3 & 0.139 & 0.177 & 0.251 \\
4 & 0.174 & 0.150 & 0.259 \\
5 & 0.235 & 0.185 & 0.259 \\
6 & 0.261 & 0.246 & 0.250 \\
7 & 0.227 & 0.272 & 0.242 \\
8 & 0.166 & 0.238 & 0.243 \\
$1^{\prime}$ & 0.769 & 0.384 & 0.333 \\
$2^{\prime}$ & 0.806 & 0.406 & 0.585 \\
$3^{\prime}$ & 0.843 & 0.473 & 0.584 \\
$4^{\prime}$ & 0.817 & 0.507 & 0.592 \\
$5^{\prime}$ & 0.852 & 0.568 & 0.592 \\
$6^{\prime}$ & 0.913 & 0.595 & 0.583 \\
$7^{\prime}$ & 0.939 & 0.560 & 0.575 \\
$8^{\prime}$ & 0.904 & 0.499 & 0.576 \\
$1^{\prime \prime}$ & 0.435 & 0.718 & 0.666 \\
$2^{\prime \prime}$ & 0.473 & 0.740 & 0.919 \\
$3^{\prime \prime}$ & 0.510 & 0.806 & 0.918 \\
$4^{\prime \prime}$ & 0.484 & 0.840 & 0.925 \\
$5^{\prime \prime}$ & 0.518 & 0.902 & 0.925 \\
$6^{\prime \prime}$ & 0.579 & 0.928 & 0.917 \\
$7^{\prime \prime}$ & 0.606 & 0.893 & 0.908 \\
$8^{\prime \prime}$ & 0.571 & 0.832 & 0.909 \\
& & & \\
& & &
\end{tabular}

structure (Figures 5A, B), while the reflections with $-h+k+l \neq 3 n$ are absent as a consequence of the rhombohedral symmetry.

The results reported suggest that $\alpha$ form samples presenting X-ray diffraction patterns similar to those of Figure $1 \mathrm{~A}$ and $\mathrm{B}$ are in modifications close to the limiting ordered and disordered ones $\left(\alpha^{\prime \prime}\right.$ and $\left.\alpha^{\prime}\right)$ and that the former can be well represented by the model of Figure 4.

It is noteworthy that the proposed model of packing of the phenyl rings in the $\alpha$ form of s-PS is very similar to that present in isotactic polystyrene, though in this case the atoms of the backbone are arranged around the threefold helical axes ${ }^{28}$ (Figure 6). This gives rise, in both cases, to a very strong (211) reflection, 

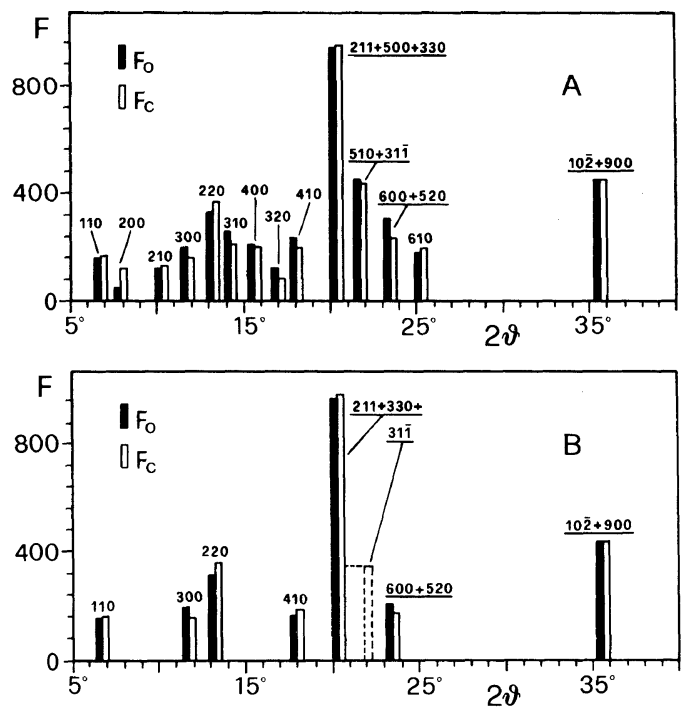

Figure 5. Observed structure factors, $F_{\mathrm{o}}$, (full rectangles) obtained from the X-ray powder diffraction spectra of Figure 1A (A) and 1B (B), corresponding to samples, close to the $\alpha^{\prime \prime}$ ordered (A) and $\alpha^{\prime}$ disordered (B) limiting modifications, respectively. Calculated structure factors, $F_{\mathrm{c}}$, (empty rectangles) for the model of Figure 4, corresponding to the space group $\mathrm{P} 3 c 1$ (A) and for the statistical model corresponding to the space group $\mathrm{R} \overline{3} c$ (B). For the $\alpha^{\prime}$ modification (part B) the structure factor for the $31 \overline{\mathrm{I}}$ reflection is presented together with the structure factors of the 211 and 330 reflections because the corresponding observed peaks are superimposed and not easy to resolve. However to make an easier comparison with part $\mathrm{A}$ of the figure, the calculated structure factor for the $31 \overline{1}$ reflection is also indicated separately as a dashed rectangle.

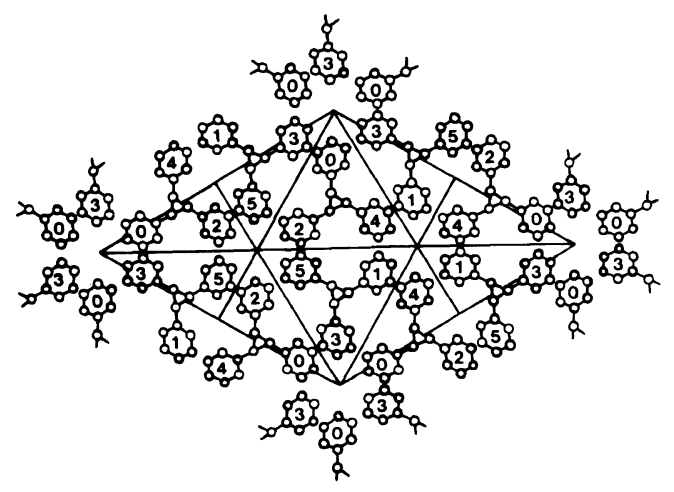

Figure 6. Model of packing of isotactic polystyrene for the R3c space group. ${ }^{28}$ Relative heights of the centers of the phenyl rings are in units $c / 6$. present in the case of s-PS at $2 \theta=20.4^{\circ}(\mathrm{Cu}-K \alpha)$. The calculated intensity of this reflection is instead very low for the previously proposed model, ${ }^{15}$ which does not present any $z$ displacement between adjacent clusters of phenyl rings.

Acknowledgements. We thank Dr. E. Albizzati and Dr. G. Giunchi of Himont Italia for their comments. The financial support of the "Ministero dell' Università e della Ricerca Scientifica e Tecnologica" of Italy and of "Progetto Finalizzato Chimica Fine e Secondaria II" of C.N.R. is gratefully ackowledged.

\section{REFERENCES}

1. N. Ishihara, T. Seimiya, M. Kuramoto, and M. Uoi, Macromolecules, 19, 2465 (1986).

2. A. Zambelli, P. Longo, C. Pellecchia, and A. Grassi, Macromolecules, 20, 2035 (1987).

3. N. Ishihara, M. Kuramoto, and M. Uoi, Macromolecules, 21, 3356 (1988).

4. Y. Chatani, Y. Fujii, Y. Shimane, and T. Ijitsu, Polym. Prepr., Jpn., (English Edition), 37, E428 (1988).

5. A. Immirzi, F. De Candia, P. Iannelli, V. Vittoria, and A. Zambelli, Makromol. Chem. Rapid Commun., 9, 761 (1988).

6. V. Vittoria, F. De Candia, P. Iannelli, and A. Immirzi, Makromol. Chem. Rapid Commun., 9, 765 (1988).

7. G. Guerra, V. M. Vitagliano, P. Corradini, and E. Albizzati, Italian Patent. 19588 (February 1989) (Himont, Inc.).

8. V. Vittoria, R. Russo, and F. De Candia, $J$. Macromol. Sci.-Phys., B28, 419 (1989).

9. G. Guerra, V. M. Vitagliano, C. De Rosa, V. Petraccone, and P. Corradini, Macromolecules, 23, 1539 (1990).

10. G. Guerra, C. De Rosa, V. M. Vitagliano, V. Petraccone, and P. Corradini, J. Polym. Sci., Polym. Phys. Ed., 29, 265 (1991).

11. G. Guerra, C. De Rosa, V. M. Vitagliano, V. Petraccone, P. Corradini, and F. E. Karasz, Polym. Commun., 32, 30 (1991).

12. C. De Rosa, M. Rapaccioulo, G. Guerra, V. Petraccone, and P. Corradini, Polymer in press.

13. M. Rapacciuolo, C. De Rosa, G. Guerra, G. Mensitieri, A. Apicella, and M.A. Del Nobile, J. Mater. Sci. Lett., 10, 1084 (1991).

14. O. Greis, T. Asano, Y. Xu, and J. Petermann, Z. Kristallogr., 182, 58 (1989).

15. O. Greis, Y. Xu, T. Asano, and J. Petermann, 


\section{De Rosa et al.}

Polymer, 30, 590 (1989).

16. P. Pradere and E. Thomas, Macromolecules, 23, 4954 (1990).

17. G. Conti, E. Santoro, L. Resconi, and G. Zerbi, Mikrochim. Acta, 1, 297 (1988).

18. R. A. Niquist, Appl. Spectrosc., 43, 440 (1989).

19. N. M. Reynolds, J. D. Savage, and S. L. Hsu, Macromolecules, 22, 2867 (1989).

20. M. Kobayashi, T. Nakaoki, and N. Ishihara, Macromolecules, 23, 78 (1990).

21. G. Guerra, P. Musto, F. E. Karasz, and W. J. MacKnight, Makromol. Chem., 191, 2111 (1990).

22. V. Vittoria, Polym. Commun., 31, 263 (1990).

23. A. Grassi, P. Longo, and G. Guerra, Makromol.
Chem. Rapid Commun., 10, 687 (1989).

24. M. A. Gomez and A. E. Tonelli, Macromolecules, 23, 3385 (1990).

25. D. C. Doherty and A. J. Hopfinger, Macromolecules, 22, 2472 (1989).

26. P. Corradini, R. Napolitano, and B. Pirozzi, Eur. Polym. J., 26, 157 (1990).

27. D. T. Cromer and J. B. Maan, Acta Crystallogr., A24, 321 (1968).

28. G. Natta and P. Corradini, Makromol. Chem., 16, 77 (1955).

29. "International Tables for X-Ray Crystallography," The Kynoch Press, Birmingham, 1952. 